\title{
Capacitación remota en competencias técnicas en el marco de la ambientalización de la Ingeniería La formación con herramientas TICs para soporte del Tele-Trabajo.
}

\author{
Remote training in technical skills \\ within the framework of the greening of Engineering \\ Training with ICT tools to support Tele-Work.
}

Cecilia Sandoval-Ruiz

cesandova@gmail.com

https://orcid.org/0000-0001-5980-292X

Universidad de Carabobo, Valencia - Venezuela

Recibido: $16 / 06 / 20$

Aceptado: 28/01/21

\section{Resumen}

Se presenta el diseño de estrategias para la capacitación remota, siguiendo criterios de ambientalización del programa de formación en ingeniería. Esto a través de una revisión conceptual de las tendencias en la ambientalización curricular. El método consistió en ensayos de actualización de los contenidos, a partir de actividades remotas. Se obtiene como resultado un nuevo enfoque en la conformación de los conceptos de eco-ingeniería y tele-trabajo. Lo que permitió concluir en la actualización de las competencias técnicas orientado al desarrollo sostenible y responsabilidad ambiental.

Palabras clave: Eco-Ingeniería, Ingeniería Digital, Tele-trabajo, capacitación remota, Universidad 4.0.

\section{Abstract}

The objective was to describe an educational experience on the construction of a Diplomat in Statistics Applied to Research with Google Classroom, Faculty of Medicine, University of Los Andes, Mérida, Venezuela, 2018-2020. Method: quantitative approach, descriptive research type, non-experimental, field design, prospective. The sample consisted of 168 participants in seven cohorts of the diplomat course. Results: 83.3\% (n $=140$ ) completed the diplomat, the average and weighted standard deviation of grades was $16.98 \pm 1.473$ points. Conclusions: the construction of the diplomat with the use of 
¿eduweb

Revista de Tecnología de Información y Comunicación en Educación • Volumen 15, Nㅜ 2. Mayo-agosto 2021

Google Classroom was effective. The educational process under the b-learning modality was fluid and allowed to overcome logistical difficulties.

Key words: Statistical Literacy, B-learning, Education.

\section{Introducción}

La población humana ha ido ocupando cada vez más espacios que pertenecen al hábitat natural de la fauna local y silvestre, en su afán de explorar territorios y recursos, alterando el equilibrio de la vida en el planeta. Todos los seres vivos (animales de todas las especies) merecen calidad de vida, paz y seguridad. Esa seguridad es alcanzada cuando el humano no interviene los ecosistemas y es responsable en causar el menor impacto ambiental, en sus actividades profesionales. La evolución del ser humano está relacionada a entender este principio y sentir empatía, respeto y amor hacia todos los seres vivos.

Actualmente, la formación profesional presenta dos retos bien definidos, el primero de ellos corresponde con la mitigación y remediación del impacto ambiental, y el segundo, accesibilidad a la formación en competencias técnicas de forma remota. Se deben asumir y sintetizar los temas en la formación del profesional, con un claro sentido de responsabilidad, específicamente en el contexto ambiental. Ante el panorama ambiental actual resulta obligatorio revisar la responsabilidad del sistema educativo y de valores profesionales, el papel que juega el currículo universitario en este modelo, así como ofrecer alternativas de solución.

En (Sabogal, 2005) se afirma que la universidad contemporánea debe responder a los desafíos que le presenta la sociedad del conocimiento, la globalización, el nuevo papel de la ciencia y la tecnología. Por su parte (Sandoval, 2016) afirma la importancia de incluir una estrategia de análisis sobre las motivaciones, ética y valores que deben estar presentes en los proyectos profesionales, como parte del proceso de formación. Igualmente, se menciona que los ingenieros deben ser capaces de reconocer, que cualquier impacto causado por el ejercicio de la profesión en un determinado entorno trasciende, todas las escalas de los sistemas y alrededores implicados (Belandria, 2011).

En (Quintana, 2015) se plantea un análisis de la ambientalización curricular, la incorporación de contenidos ambientales en el programa universitario y revela conexiones entre la sociedad, el diseño curricular, la formación ambiental de docentes y por último potencialidades e implicaciones de las TIC para la ambientalización del proceso formativo. Por otra parte (Mora, 2011) plantea la necesidad de considerar indicadores de un modelo de inclusión de dimensiones ambientales en el currículo de educación superior. En este orden de ideas, se consideran los indicadores que permitan medir la eficiencia de los programas de formación y actualización en competencias técnicas para el tele-trabajo en áreas de ingeniería, cumpliendo con criterios de bajo impacto ambiental. A modo de complementar las competencias profesionales, con un 
sentido de responsabilidad sobre el efecto de las acciones profesionales sobre la flora, fauna, espacios, y recursos renovables.

El objetivo de este trabajo es desarrollar una propuesta enfocada en un modelo de desarrollo sostenible planteada por (Barrón, 2010) que le brinde herramientas y competencias al profesional bajo criterios de análisis y estimación del impacto ambiental de los proyectos, en el marco del ejercicio profesional. En (Mora, 2012) se menciona que es necesario facilitar a los estudiantes universitarios, competencias no solo científicotécnicas sino también sociales y éticas coherentes con un desarrollo humano sostenible. Las competencias profesionales han sido entendidas como el conjunto de conocimientos, habilidades, destrezas y actitudes. La adquisición de determinadas competencias sería uno de los objetivos del proceso formativo, dotando en este sentido de utilidad a la adquisición de conocimientos en función de un contexto social y laboral específico. Las competencias ambientales entendidas como el resultado final de un proceso formativo, están dirigidas en primera instancia a la comprensión de la relación medioambiente sociedad; luego a la adquisición de conocimientos y la creación de habilidades, destrezas para la solución de problemas en un entorno marcado por la condición ambiental (Quintana, 2015).

En (Mora, 2011) se afirma que los problemas ambientales son principalmente problemas de conocimiento, del modelo de desarrollo y de la educación global y local. Esta concepción permite encontrar una solución a partir de nuevos enfoques en la matriz curricular, para dirigir el conocimiento hacia un modelo de desarrollo respetuoso con el medio ambiente. Los objetivos del currículo están enmarcados en orientar de manera continua el proceso de formación integral del talento humano, profesional y especialistas, para promover su actualización y mejoramiento conforme a las necesidades tecnológicas y progreso científico. Al momento de definir los valores como eje del programa de formación, se debe considerar la responsabilidad, empatía con el eco-sistema, establecer motivaciones con el sentido de la vida, decisiones profesionales con criterios sostenibles, consumo ético, valorar la vida, respecto a los seres vivos y la naturaleza.

\section{Problemática o Retos Actuales}

Los retos que se presentan actualmente están orientados a la necesidad de fomentar espacios de actualización de profesionales en áreas técnicas para soporte del teletrabajo desde el punto de vista de la dinámica laboral de los nuevos tiempo, así como estrategia de responsabilidad social, a fin de garantizar seguridad y ambientes más flexibles para desempeñar tareas de ingeniería de forma remota. Además de la necesidad de reorientar los métodos de enseñanza hacia la conscientización de los estudiantes en eco-ingeniería, que aborde la problemática del estudio de impacto ambiental de los proyectos y mejoras en la calidad de vida de la fauna, los ecosistemas y los urbanismos, donde se valore la naturaleza como primer criterio de desarrollo y se mantengan principios de eficiencia energética, sostenibilidad y responsabilidad socioambiental. Para el diseño de sistemas regenerativos que se encuentren en armonía con el entorno.

12 Capacitación remota en competencias técnicas en el marco de la ambientalización de la Ingeniería. La formación 


\section{Fundamentos}

Uno de los conceptos para la formación práctica del personal técnico y de ingeniería, básicos para el soporte de las actividades corresponde a los laboratorio virtuales 0 remotos, por sus ventajas (Vergara, 2020). Por otra parte, para lograr estos objetivos los programas de formación deben cumplir con actividades en base a modelos pedagógicos que manifiesten una integración de lo cognitivo, las competencias y los modos de actuación profesional (Carbonell, 2004).

Enseñanza de base dirigida a una ambientalización de los programas de formación, con el objetivo de incluir en los esquemas formativos de los mismos los conceptos e instrumentos que deberían permitirles comprender y apreciar el medio ambiente y su complejidad, entender su relación en la actividad humana e integrar el factor ambiental en la actividad profesional, apoyado en especialistas en ciencias ambientales, o en ingeniería y gestión ambiental. Actualizaciones en talento humano a nivel técnico, enseñanza a nivel de master, orientada a la especialización profesional en una temática ambiental concreta, e investigaciones doctorales.

Es necesario establecer normas para este nuevo modelo, adaptados al nivel de formación/actualización, programas y estrategias propia del docente en el diseño de la asignatura, prácticas y reflexiones de construcción de valores. Al momento de construir los indicadores se puede tomar como referencia el resumen presentado en (Mora, 2007). Donde es válido destacar que cada uno de los indicadores debe ser contextualizado a la realidad actual, considerando los elementos de la Tabla 1.

Tabla 1. Indicadores de sostenibilidad en el currículo

\begin{tabular}{|c|c|c|c|c|c|}
\hline Profesión & 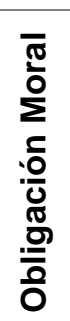 & $\begin{array}{l}\frac{0}{0} \\
\frac{\pi}{0} \\
\overline{0} \\
\frac{0}{\pi} \\
0 \\
0\end{array}$ & 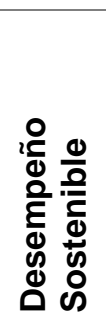 & 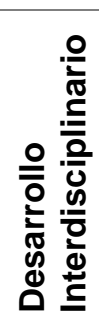 & $\begin{array}{l}\frac{0}{0} \\
: \frac{0}{0} \\
\frac{0}{\mathbb{J}} \\
\frac{.0}{0} \\
\frac{0}{4} \\
\end{array}$ \\
\hline Ingeniería en Fuentes de Energías Renovables & $\mathrm{x}$ & $\mathrm{x}$ & $\mathrm{x}$ & $\mathrm{x}$ & $\mathrm{x}$ \\
\hline Eco-Educación & $\mathrm{x}$ & $\mathrm{x}$ & $\mathrm{x}$ & $\mathrm{x}$ & $x$ \\
\hline Tecni-Agronomía Sustentable & $\mathrm{x}$ & & $\mathrm{x}$ & $\mathrm{x}$ & $x$ \\
\hline Gestión de Recursos Renovables & $\mathrm{x}$ & $\mathrm{x}$ & $\mathrm{x}$ & $\mathrm{x}$ & $\mathrm{x}$ \\
\hline Arquitectura Sostenible & $\mathrm{x}$ & $\mathrm{x}$ & $\mathrm{x}$ & $\mathrm{x}$ & $\mathrm{x}$ \\
\hline
\end{tabular}

Cecilia Sandoval-Ruiz.

El actual currículo universitario debe actualizar los términos manejo ecológico, como parte fundamental del contexto educacional. Siendo un elemento común a cada una de las disciplinas: Ingeniería en alimentos (Sandoval, et. al., 2018), Arquitectura Sostenible (Sandoval, 2015), aplicaciones matemáticas (Sandoval, 2020d, 2021b). 
Las investigaciones recientes presentan una tendencia en materia de incorporación de energías renovables, a fin de garantizar los objetivos de desarrollo sostenible - ODS, se plantea la incorporación de un espacio de investigación como lo es un laboratorio para la educación en energías renovables y aspectos en esta materia. Para dar respuesta efectiva a todas ellas es preciso construir capacidades e instituciones destinadas a generar e implementar estrategias, en el sector laboral y académico, con una visión transformadora hacia la sostenibilidad económica, social y ambiental. Considerando todo lo anterior, (Pérez, 2005) plantea una educación para cambiar la sociedad, una educación total que contribuya a la mejora de la calidad de vida de las personas y el medio ambiente. Las universidades y las instituciones de capacitación, pueden ser un interesante vehículo para favorecer el desarrollo sostenible. A tales propósitos responden iniciativas como las de la Organización Internacional de Universidades por el Desarrollo Sostenible y el Medio Ambiente, al plantearse objetivos con lo que se busca incluir temáticas ambientales, dimensiones del desarrollo sostenible, medio ambiente y desarrollo sostenible y construcción de metodologías de evaluación basadas en indicadores de sostenibilidad.

\section{Currículo y Tecnología de la Información}

En (Sandoval, 2017) se plantean estrategias didácticas haciendo uso de tecnología de la información y comunicaciones, como alternativa a la planificación de aula, que viene a complementar el desempeño en tecnologías sostenibles. En (Sandoval, 2014) se presentan avances de aplicaciones web, se enunció una propuesta de un entorno colaborativo de Investigación $\mathrm{ECIC}$, que cuente con las características requeridas para optimizar el proceso de producción científica, brindando un soporte para la investigación a distancia, entre grupos colaborativos y con instalaciones y equipos remotos, que puedan ser tele-operados desde la plataforma web, el diseño conceptual de la estrategia de ambientalización se presenta en la Figura 1.

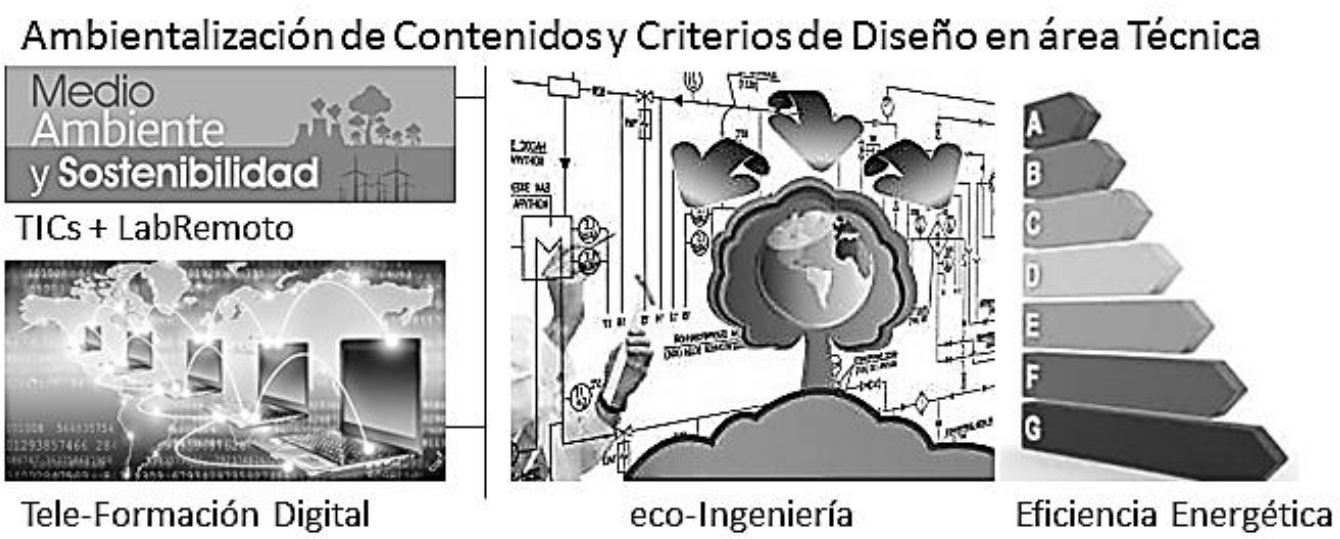

Figura 1. Diseño Conceptual de la Estrategia. 
El diseño curricular maneja tópicos de eficiencia energética, diseño con estimación de impacto ambiental y manejo remoto de las actividades técnicas, para lo que se han diseñado módulos específicos.

\section{Metodología}

El método consistió en diagnosticar las necesidades de actualización del modelo vigente, a fin de desarrollar una propuesta bajo criterios de sostenibilidad y responsabilidad ambiental, que demandan la actualización en campo de los profesionales para ciertas tareas específicas, como en el caso de ingeniería solar, eólica, entre otras. De la misma manera, el campus universitario debe reflejar la aplicación de conceptos ecológicos que permitan afianzar estos conocimientos, haciéndose familiares para el estudiante y el desarrollo de sus investigaciones. Tratando de crear un acercamiento entre los aspectos propios de la matriz curricular con estos tópicos cada vez más vigentes e importantes, a fin de egresar profesionales competitivos en ambientes internacionales.

Por otra parte, la revisión de los indicadores está basada en preguntas de índole general, sobre la materia a partir de las carreras universitarias vigentes, así como la propuesta de carreras novedosas enfocadas en nuevos objetivos, ajustados a las necesidades de protección ambiental, formando los criterios desde la reflexión del participante (ver Tabla 2).

Tabla 2. Preguntas de formación de criterios ambientales.

a. ¿Qué efectos presenta el ejercicio profesional sobre el ambiente?

b. ¿Se generan residuos contaminantes en el proceso de producción / competencia de la profesión?

c. ¿Qué impacto presenta sobre la diversidad biológica?

d. ¿Se encuentra basada en energías renovables o convencionales con emisiones de CO2?

e. ¿Se promueve el agotamiento de recursos naturales en la producción de insumos?

f. ¿Estimación de impacto ambiental de los proyectos, investigaciones y desarrollos?

En términos de aplicación de tecnología para la capacitación remota, orientada al teletrabajo en el área técnica, se seleccionó el diseño de laboratorios remotos, siguiendo el concepto de Industria 4.0 aplicada a la migración energética hacia energías renovables no convencionales. El talento humano tiene acceso de forma remota, sin trasladarse a universidades, plantas de procesos industriales, etc., a los ambientes reales a través de internet, para soporte de este sector industrial, con configuración a distancia de sus procesos.

\section{Resultados}

Se desarrolló una matriz comparativa, a fin de establecer identificadores por carreras, como se presenta en la Tabla 3. 
Tabla 3. Identificadores por Carrera Universitaria

\begin{tabular}{ll}
\hline Carreras & Estrategias Sostenibles \\
\hline Ingeniería & Tipo de Energía Renovables, Estudio de Impacto Ambiental \\
\hline Electrónica & Hardware Reconfigurable vs. Obsolescencia Programada \\
\hline Industrial & Re-utilizabilidad de Materia Prima, Reciclaje y Sub-Productos \\
\hline Agronomía & Modelo de productos orgánicos. \\
\hline Medicina & Tratamiento de Productos Descartables \\
\hline Forestal & Gestión de Recursos Renovables \\
\hline
\end{tabular}

Así mismo el diseño de estimadores de eficiencia energética, impacto ambiental, consumo de recursos y su reinserción a la matriz productiva, pueden ser mecanismos para soportar la nueva visión de enseñanza universitaria, tal como se presenta en la Tabla 4.

Tabla 4. Estrategias de Tele-capacitación en competencias técnicas

\begin{tabular}{|c|c|}
\hline Estrategia & Descripción de la Estrategia \\
\hline $\begin{array}{ll}\text { Laboratorio } & \text { Remoto } \\
\text { (Rondón, 2010) }\end{array}$ & $\begin{array}{l}\text { Prácticas para el diseño de hardware de electrónica reconfigurable, a } \\
\text { través de una plataforma remota. }\end{array}$ \\
\hline $\begin{array}{l}\text { Robótica Tele-op. } \\
\text { (Valero-Moro, 2017) }\end{array}$ & $\begin{array}{l}\text { Teleoperación de mecanismos robóticos en el laboratorio para soporte de } \\
\text { actividades en tele-trabajo en condiciones seguras. }\end{array}$ \\
\hline $\begin{array}{l}\text { Lab. en Tecnologías } \\
\text { Sostenibles (Sandoval, } \\
\text { 2016) }\end{array}$ & $\begin{array}{l}\text { El diseño de plataformas para la enseñanza de tecnologías sostenibles, } \\
\text { ofrecen una herramienta que facilite la adaptación del programa, así como } \\
\text { los módulos prácticos (Sandoval, 2013) }\end{array}$ \\
\hline \multirow{4}{*}{$\begin{array}{l}\text { Propuesta } \\
\text { eco-Ingeniería } \\
\text { (Sandooval, 2021c) }\end{array}$} & VNC para establecer conexión remota con lab-servidor \\
\hline & IDE de desarrollado para síntesis de hardware en VHDL \\
\hline & Raspberry Pi para control FPGA electrónica recon \\
\hline & Análisis de eficiencia energética de los diseños en línea \\
\hline $\begin{array}{l}\text { FV Reconfig } \\
\text { (Sandoval, 2020a,b }\end{array}$ & \multirow{2}{*}{$\begin{array}{l}\text { Plataforma de soporte con R-IEDs para la configuración de los arreglos } \\
\text { fotovoltaicos y etapas, extrapolados a esquemas de energías renovables } \\
\text { ERNC y sistemas de ingeniería sostenible, con monitoreo on-line de } \\
\text { variable ambientales. }\end{array}$} \\
\hline $\begin{array}{l}\text { Smart Energy (Sandoval, } \\
\text { 2020c) }\end{array}$ & \\
\hline $\begin{array}{l}\text { Eco-Parque Universit. } \\
\text { (Steckler, 2018) }\end{array}$ & $\begin{array}{l}\text { parque diseñado para implementar e } \\
\text { áctica y protección a la fauna. }\end{array}$ \\
\hline
\end{tabular}

Comprende un compendio de técnicas innovadoras para el desarrollo de la capacitación remota en Ingeniería Sostenible.

Esto partiendo de las necesidades de formación en energías renovables para la adquisición de competencias para el emprendimiento (CEPAL, 2017), sobre la que se diseñan los módulos presentados en la Tabla 5. 


\section{Geduweb}

Revista de Tecnología de Información y Comunicación en Educación • Volumen 15, Nㅜ 2. Mayo-agosto 2021

Tabla 5. Módulos de Actualización en ERNC

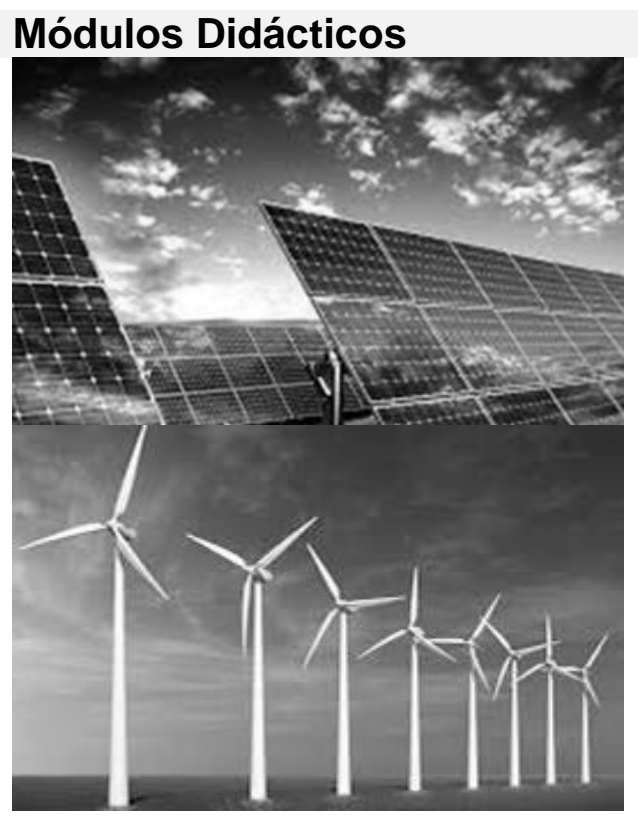

\section{Objetivos}

Fotovoltaica. Establecer prácticas de configuración remota, reconocer las ventajas con sistemas de energía convencional. Proyectos de MPPT y optimización.

Eólica. Estudio a nivel de simulación, con herramientas software. CFD Simulación computacional de dinámica de fluidos, análisis de eficiencia.

Se requiere la creación de programas estratégicos de apoyo a la investigación en disciplinas asociadas a la sostenibilidad, que faciliten y potencien estas actividades en las unidades de capacitación, de forma práctica. Con lo que se puede desarrollar un programa adaptado a los retos propios de las necesidades y potencialidades locales, ajustándose a la dinámica educacional, como se expone en las características del currículo señaladas por (Velásquez, 2009).

\section{Estrategias para el diseño curricular con enfoque ambiental}

En primer lugar, la adaptación curricular, según (Pérez, 2005) consiste en la incorporación de la dimensión ambiental en los contenidos formativos. En el marco del currículo universitario se plantea colocar un objetivo por asignatura relacionados con el impacto ambiental del contenido estudiado, de manera de asociar un sentido de monitoreo permanente, por parte del estudiante, de los efectos y consecuencias de cada actividad y decisión profesional. Esto puede lograr un cambio de paradigma, en la proyección de las carreras universitarias, haciéndolas más eco-sociales, pensadas en el beneficio de todos los actores, entre estos la naturaleza y recursos ambientales.

Seguido de una estrategia de gestión ambiental sostenible, las universidades como organizaciones generan una serie de impactos sobre el medio y deben planificar actuaciones correctoras que eviten o minimicen esos impactos, mediante la implantación de los correspondientes sistemas de gestión ambiental y la planificación a corto, medio y largo plazo, en el desarrollo de compromisos ambientales; en la gestión de recursos 
como el agua, los residuos, aumento de la autonomía por la filosofía de diseño, eficiencia energética, uso de energías renovables y ahorro energético.

Una universidad sostenible tendrá un impacto positivo en el ambiente y en los individuos en proceso de formación. Con esta propuesta se logra dar un giro al modelo de educación con fines de proteger el ambiente, desde los principios éticos y valores del profesional. Más allá de la tecnificación del conocimiento, se promueve el pensamiento reflexivo y una consciencia educacional de respeto y responsabilidad ambiental. Haciendo uso de las nuevas tecnologías para el diseño de un modelo curricular más competente y con aportes significativos en la perspectiva de las profesiones en el futuro.

Finalmente, una estrategia de educación y participación ambiental, parte de la idea de que la ambientalización del programa, oportunidades para que los estudiantes incorporen en sus estilos de vida criterios de sostenibilidad (Sandoval, 2018) y en sus códigos profesionales, principios y fundamentos inspirados en las buenas prácticas proambientales. La educación en valores prácticos, tratará de enseñar los conceptos teóricos, y prácticos, que permita al estudiante ser agente transformador. Este aspecto puede estar contemplado en el diseño de actividades y proyectos dentro del currículo universitario.

\section{Conclusiones}

Gracias al modelo acá propuesto, se puede aportar un novedoso enfoque, en el cual se integra al compendio de criterios de índole social, tecnológica y ambientalización del programa de formación. Este modelo comprende objetivos de estimación del impacto ambiental dentro de los proyectos, prácticas experimentales y actividades remotas de laboratorio (Sandoval, 2021a). La tele-educación debe llevar un mensaje de sostenibilidad, avanzar en temas de protección ambiental y eficiencia.

De esta manera, la ambientalización curricular e incorporación de temas de actividades Sostenible en los programas de formación de competencias, permitirán crear conciencia desde los espacios de educación, en valores de respeto y responsabilidad con el ambiente, protección del hábitat de la fauna urbana y silvestre y los recursos naturales del planeta, con prácticas como huertas urbanas orgánicas, alimentación vegetariana (Sandoval, 2017), como estrategia de seguridad alimentaria, reciclaje, tele-trabajo, energías renovables distribuidas, tiempos de recuperación/descanso, entre muchas materias a incluir en la formación ambiental, pensadas en mejorar la calidad de vida.

Integrar algunos tópicos de educación acerca de la forma responsable de interactuar con la fauna, qué alimentos son apropiados, sus características, la no intervención en espacios silvestres que puedan alterar equilibrio de la fauna y su entorno, respetar las especies locales, todos estos factores muchas veces no son informados sino a especialistas, causando errors por desconocimiento que alteran el bienestar de los ecosistemas. Con estas estrategias se pretende incorporar en las directrices colectivas 
de la academia universitaria, la evaluación de los efectos directos e indirectos de las actividades de profesionales más conscientes y sensibilizados.

Otro aspecto corresponde al enfoque transversal, entre formación de valores, capacitación en herramientas remotas y criterios éticos, que los proyectos de investigación, cuentan con una dimensión de factibilidad técnica-ambiental, que puede ser adaptada para independizar las funciones de la tele-ingeniería. Una alternativa con alto potencial corresponde al uso de TICs en el modelo del currículo universitario, que permita integrar los criterios y formas de auditoria del enfoque sostenible.

Se han planteado estrategias innovadoras, a fin de crear un ambiente de desarrollo sostenible y una plataforma de servicio para actualización de telento humano, enmarcado en los aspectos formativos y participativos del estudiante. Se necesita complementar con valores de eco-responsabilidad, siendo este un aspecto característico del nuevo modelo de desarrollo. Profesionales más conscientes y comprometidos con el tratamiento racional de los recursos no renovables, así como respetar los ciclos de regeneración natural de los recursos renovables.

\section{Referencias}

Barrón, Á., Ferrer-Balas, D., \& Navarrete Salvador, A. (2010). Sostenibilización curricular en las universidades españolas. ¿ha llegado la hora de actuar?.

Belandria, J. (2011). Importancia de la formación humanística, ecológica, social y ética en los estudios de ingeniería. Revista Ciencia e Ingeniería, Enseñanza de la Ingeniería, 17-24.

Carbonell, E. A. F., Machado, J. L., \& Conde, A. P. (2004). Universidad y Desarrollo Sostenible. Pedagogía Universitaria, 9(3).

CEPAL. (2017). Perspectivas económicas de América Latina 2017. Retrieved from https://www.oecd.org/dev/americas/E-book_LEO2017_SP.pdf

Mora Penagos, W. M. (2007). Respuesta de la universidad a los problemas socio ambientales: la ambientalización del currículo en la educación superior. Revista Investigación en la Escuela, 63, 65-76.

Mora Penagos, W. M. (2011). La inclusión de la dimensión ambiental en la educación superior: un estudio de caso en la Facultad de Medio Ambiente de la Universidad Ditistral en Bogotá.

Mora, William (2012) "Ambientalización curricular en la educación superior: un estudio cualitativo de las ideas del profesorado" en Revista de currículum y formación del profesorado, España: Vol.16, No.2.

Pérez, J. G., \& Dulzaides, A. G. (2005). Ambientalizar la universidad: un reto institucional para el aseguramiento de la calidad en los ámbitos curriculares y de la gestión. Revista Iberoamericana de Educación, 36(7), 1-14. https://rieoei.org/historico/deloslectores/890Gutierrez.PDF

Quintana, G. E., \& Mateos, J. E. G. (2015). ¿ Incluir contenidos ambientales o formar con una perspectiva ambiental?. RIPS: Revista de Investigaciones Políticas y 
Sociológicas, 13(2).

Rondón, J., \& Sandoval, C. (2010). Diseño de un co-laboratorio remoto basado en programación modular de dispositivos VHDL aplicado a telecomunicaciones. Facultad de Ingeniería UCV, 25(2), 7-12. Retrieved from http://www.scielo.org.ve/pdf/rfiucv/v25n2/art02.pdf

Sabogal, L. M. D. B. (2005). Reflexión sobre el currículo universitario desde la teoría discursiva de la educación. Revista ieRed: Revista Electrónica de la Red de Investigación Educativa, 1(3), 5.

Sandoval-Ruiz, C. (2021a). Laboratorio Móvil para Optimización de Sistemas de Energías Renovables y Aplicaciones Ambientales. Ciencia e Ingeniería, 42(2), 169178.

Sandoval-Ruiz, C. (2021b). Fractal Mathematical over Extended Finite Fields $F p[x] /(f(x))$. Proyecciones Journal of Mathematics, Vol. 40(3), 731-742. doi.org/10.22199/isnn.0717-6279-4322.

Sandoval-Ruiz, C. (2021c). Modelo de Sistemas Inteligentes Regenerativos para la protección de Ecosistemas, Flora y Fauna. Universidad, Ciencia y Tecnología, 25(110).

Sandoval-Ruiz, C. E. (2020a). Arreglos fotovoltaicos inteligentes con modelo LFSRreconfigurable. Revista Ingeniería, 30(2), 32-61. DOI 10.15517/RI.V30I2.39484.

Sandoval-Ruiz, C. E. (2020b). Proyecto Cometa Solar-CS para Optimización de Sistemas Fotovoltaicos. Universidad Ciencia y Tecnología, 24(100), 74-87.

Sandoval-Ruiz, C. (2020c). LFSR-Fractal ANN Model applied in R-IEDs for Smart Energy. IEEE Latin America Transactions, 18(04), 677-686.

Sandoval-Ruiz, C. (2020d). C."Operador matemático LFC (n,k) en campos finitos basado en concatenación fractal para GF (2m)-Extendido". Ciencia e Ingeniería, 41(2), 197-204.

Sandoval-Ruiz, C. (2020e). Arreglo Inteligente de Concentración Solar FV para MPPT usando Tecnología FPGA. Revista Técnica Universidad del Zulia, 4(3), 122-133. doi.org/10.22209/rt.v43n3a02.

Sandoval-Ruiz, C. E., \& Ruiz-Díaz, E. (2018). Optimizador de eco-productos de origen vegetal aplicando control neuronal en VHDL. Revista Agrollanía. Vol. 15, 58-64.

Sandoval, C. (2017). Estrategias de aprendizaje colaborativo, a través de las TICs y grupo de investigación. Revista de Tecnología de Información y Comunicación en Educación - Eduweb, Univ. Carabobo, 11(2), 99-112.

Sandoval Ruiz, C. (2016). Plataforma de Gestión, Investigación y Formación en Tecnologías Sostenibles, para soporte de un Laboratorio Remoto. Revista Eduweb, Universidad de Carabobo, 10(1), 79-92.

Sandoval-Ruiz, C. (2015). Sistema Eco-Adaptativo integrado en elementos arquitectónicos con tecnología sostenible. Revista Electrónica Científica Perspectiva, 8(4), 96-109. https://issuu.com/recperspectiva/docs/rec8/96

Sandoval-Ruiz, C. (2014). Entorno Colaborativo de Investigación científica - ECIC: Propuesta basada en Web-Lab y redes asesorías. Revista de Tecnología de Información Y Comunicación En Educación Eduweb, 8(2), 69-82. http://servicio.bc.uc.edu.ve/educacion/eduweb/v8n2/art06.pdf

Sandoval Ruiz, C. (2013). Diseño conceptual de un módulo híbrido para Generación 
eléctrica. In VIII Congreso Nacional y 2do Congreso Internacional de Investigación Universidad de Carabobo.

Velásquez S, J. A. (2009). La transversalidad como posibilidad curricular desde la educación ambiental. Revista Latinoamericana de Estudios Educativos (Colombia), 5(2), 29-44.

Steckler, D. J., Navaa, C., Duartea, J., Zambranoa, J. J., \& Sandoval-Ruiz, C. E. Design of Neural Networks on microcontrollers, applied in functional modules for an ecopark.

Valero-Moro, J., Bonilla-Turmero, Y., \& Sandoval-Ruiz, C. (2017). Estación tele-operada de robótica móvil, para el laboratorio de micro-controladores. Universidad, Ciencia Y Tecnología, 21(83), 69-75.

Vergara, D. (2020). Imposición de los Laboratorios Virtuales en la Educación del Siglo XXI. Revista Eduweb, Universidad de Carabobo, 13(2), 119-128. 